\title{
ENERGY OF THE TAUB COSMOLOGICAL SOLUTION
}

\author{
PAUL HALPERN \\ Department of Mathematics, Physics and Computer Science, \\ University of the Sciences in Philadelphia, 600 S. 43rd St., \\ Philadelphia, PA. 19104, USA \\ p.halper@usip.edu
}

\begin{abstract}
We calculate the total energy of Taub's 1951 exact solution for a Bianchi typeIX geometry using several different energy-localization procedures, including the prescriptions of Einstein, Papapetrou, Landau-Lifshitz and Møller. We compare these results to those for other anisotropic geometries, and comment on their relationship to Rosen's conjecture about the total energy of the universe.

Keywords: Taub solution; Mixmaster universe; energy-momentum complex.

PACS numbers: 04.20.Cv, 04.20.Jb, 98.80.Jk
\end{abstract}

\section{Introduction}

A longstanding question in general relativity is whether gravitational energy can be localized and, if so, what measure to use. Einstein proposed the first energy-momentum complex in an attempt to define the local distribution of energy and momentum [1]. Einstein's measure is non-tensorial and asymmetric in its indices. These issues have inspired a number of alternative measures, including prescriptions by Møller [2], Tolman [3], Papapetrou [4], Landau and Lifshitz [5], Weinberg [6] and so forth. By in large, these formulations depend on the coordinate-system used. For example, Einstein's prescription favors quasiCartesian coordinates, defined as those coordinates $x^{i}$ for which the metric components $g_{\mu \nu}$ converge sufficiently rapidly toward the constant components of the diagonal Minkowski metric $\eta_{\mu \nu}$. Some of these complexes have other restrictions.

Such limitations have led some researchers to discount the importance of localized measures. However, in 1990 Bondi proved that energy must be localizable, at least in principle [7]. The same year, Virbhadra demonstrated that these complexes, though non-tensors, yield credible, consistent results when applied to the same spacetime geometries [8]. In 1993, Rosen and Virbhadra employed the Einstein prescription and found reasonable results for the energy and momentum of cylindrical gravitational waves [9]. Later, Virbhadra derived similar results using Tolman's procedure and the measure proposed by Landau and Lifshitz [10]. Then in 1996, Aguirregabiria, Chamorro and Virbhadra found that for any metric of the Kerr-Schild class (including the Schwarzschild, ReissnerNordström, Kerr and Vaidya metrics, for example.), various energy-momentum 
prescriptions yielded reasonable and consistent results [11]. Of these, Virbhadra found that the Einstein prescription offered the greatest consistency [12]. These findings have stimulated a number of investigations attempting to extend such methods to other classes of metrics [13].

Much work has been done, for example, in using various measures to determine the energy distributions of assorted generalizations of black holes. Chamorro and Virbhardra [14] as well as Xulu [15] have examined the energy distribution of charged black holes with a dilaton field. Radinschi and Yang [16], Vagenas [17], Gad [18] and Xulu [19] have each looked at the energy distribution of stringy black holes. Vagenas has also examined black holes in $2+1$ dimensions $[20,21]$ and applied Møller's prescription to the dyadosphere of a Reissner-Nordström black hole [22]. The energy distribution of black plane solutions has also been investigated [23]. Other researchers have looked at the energy distribution for the case of teleparallel gravity [24], for a stationary beam of light [25, 26], for a radiating charged particle [27] and for a host of other metrics.

Cosmological solutions are another area of considerable interest, stimulated by a conjecture made by Rosen in 1994 that the total energy of the universe, presuming it has a closed geometry, is zero [28]. Applying the Einstein energymomentum complex to a closed Friedmann-Robertson-Walker (FRW) cosmology, Rosen determined its total energy to be indeed zero. Johri, Kalligas, Singh and Everitt later reached similar conclusions by applying the prescription of Landau and Lifshitz [29].

One possible implication of Rosen's conjecture is a hypothesis proposed by Cooperstock that the energy distribution is non-zero only in regions where the stress-energy tensor $T_{\mu \nu}$ is non-zero [30]. This implies that gravitational waves traveling through a vacuum would carry only information content, not energy, and thus would be fundamentally different from other forms of radiation.

Researchers have sought to extend these results to more general cosmologies. The isotropic FRW model is far from the most general cosmological solution, and therefore would not, for example, represent a chaotic very early stage of the universe. The simplest anisotropic model is the Kasner solution, possessing a Bianchi type I geometry. Several researchers have investigated the total energy of this model using a variety of measures, and have found the result to be identically zero for any closed region, consistent with Rosen's conjecture $[31,32,33,34,35]$. In addition to the standard vacuum case, Salti looked at the viscous Kasner universe in teleparallel gravity [36] using the Einstein and Landau-Lifshitz formulations and found the total energy and momentum to be identically zero. Generalizing these findings to another anisotropic cosmology, Radinschi examined the energy-momentum of the Bianchi type VIO universe using a number of different measures, finding that all the components vanish 
[37].

\section{The Taub Cosmological Solution}

Amongst homogenous cosmologies, the metric that yields the most complex anisotropic behavior possesses a Bianchi type IX spatial geometry. The oneforms associated with the spatial part of this metric possess the Lie algebra associated with one of the normal subgroups of the group of motion of a threedimensional sphere.

As Belinski, Khalatnikov and Lifshitz demonstrated in 1970, in evolving the vacuum case backward in time toward its initial singularity, the behavior of its three scale factors resembles a sequence of transitions between Kasner-like epochs [38]. In using Hamiltonian methods to analyze this behavior, Misner dubbed it the "Mixmaster universe," because of its special mixing properties [39]. Barrow later demonstrated that the vacuum Bianchi type IX solution exhibits a form of deterministic chaos [40].

If one constrains two of the three scale factors to be equal, one obtains an exact, non-chaotic solution, first identified by Taub in 1951. The Taub metric can be expressed in a holonomic coordinate system as:

$$
\begin{aligned}
d s^{2} & =\gamma(t) d t^{2}-R(t) d x^{2}-\left(R(t) \sin ^{2}(x)+S(t) \cos ^{2}(x)\right) d y^{2} \\
& -2 S(t) \cos (x) d y d z-S(t) d z^{2}
\end{aligned}
$$

with $x, y$ and $z$ corresponding to the Euler angles of three-dimensional rotation, and having the ranges:

$$
0 \leq x \leq \pi ; \quad 0 \leq y \leq 4 \pi ; \quad 0 \leq z \leq 2 \pi
$$

As Taub demonstrated, to satisfy the vacuum Einstein equations the scale factors are constrained to be:

$$
\begin{aligned}
R(t) & =\frac{k \cosh (k t+\alpha)}{4 \cosh ^{2}\left(\frac{k t+\beta}{2}\right)} \\
S(t) & =\frac{k}{\cosh (k t+\alpha)}
\end{aligned}
$$

where $\alpha, \beta$ and $k$ are constants and with:

$$
\gamma(t)=R(t)^{2} S(t)
$$


One reason the Taub solution is of interest is that in 1968, Misner and Taub [41] proved that it represents the spacetime region bounded by the horizon of the NUT solution found by Newman, Unti and Tamburino [42]. Joined together, these constitute the Taub-NUT spacetime.

\section{Finding the Energy Using Einstein's Energy-Momentum Complex}

It is interesting to calculate the total energy of the Taub cosmology using several different measures in order to make a comparison of the results. We

begin with the Einstein prescription, for which the energy-momentum complex is defined as:

$$
\theta_{i}^{k}=\frac{1}{16 \pi} H_{i}^{k l}, l
$$

with the superpotentials $H_{i}{ }^{k l}$ given by:

$$
H_{i}^{k l}=\frac{g_{i n}}{\sqrt{-g}}\left[-g\left(g^{k n} g^{l m}-g^{l n} g^{k m}\right)\right]_{, m}
$$

which possesses the antisymmetric property that:

$$
H_{i}{ }^{k l}=-H_{i}^{l k}
$$

Substituting into (7) the metric components of the Taub solution (1-5) we find that the only non-zero components of $H_{i}{ }^{k l}$ are:

$$
H_{0}{ }^{01}=-H_{0}{ }^{10}=-\frac{k^{2}}{8} \cos (x) \operatorname{sech}^{2}\left(\frac{k t+\beta}{2}\right)
$$

Therefore, from (6) the only non-zero components of $\theta_{i}{ }^{k}$ are:

$$
\begin{aligned}
\theta_{0}{ }^{0} & =\frac{k^{2}}{128 \pi} \sin (x) \operatorname{sech}^{2}\left(\frac{k t+\beta}{2}\right) \\
\theta_{0}{ }^{1} & =-\frac{k^{3}}{128 \pi} \cos (x) \operatorname{sech}^{2}\left(\frac{k t+\beta}{2}\right) \tanh \left(\frac{k t+\beta}{2}\right)
\end{aligned}
$$

In general, the energy-momentum components can be found by the volume integral:

$$
P_{i}=\iiint \theta_{i}^{0} d x^{1} d x^{2} d x^{3}
$$

Thus, if we substitute the coordinate ranges (2) we can write the total energy as: 


$$
P_{0}=\int_{0}^{\pi} \int_{0}^{4 \pi} \int_{0}^{2 \pi} \theta_{0}{ }^{0} d x d y d z
$$

Evaluating this integral for (10), we obtain an expression for the total energy of the Taub solution:

$$
E=P_{0}=\frac{\pi k^{2}}{8} \operatorname{sech}^{2}\left(\frac{k t+\beta}{2}\right)
$$

\section{Following Papapetrou's Procedure}

Unlike Einstein's prescription, the energy-momentum complex of Papapetrou has the advantage of being symmetric in its indices. Hence it allows local conservation laws to be well-defined.

The Papapetrou energy-momentum complex is defined as:

$$
\begin{gathered}
\Omega^{i k}=\frac{1}{16 \pi} N^{i k a b}, a b \\
P_{i}=\iiint \Omega^{i 0} d x^{1} d x^{2} d x^{3}
\end{gathered}
$$

where the functions $N^{i k a b}$ are given by:

$$
N^{i k a b}=\sqrt{-g}\left[g^{i k} \eta^{a b}-g^{i a} \eta^{k b}+g^{a b} \eta^{i k}-g^{k b} \eta^{i a}\right]
$$

and the $\eta^{a b}$ terms represent the components of a Minkowski metric of signature -2 .

Inserting the metric components of the Taub solution (1-5) into expression (17), we obtain the following relevant non-zero components of $N^{i k a b}$ :

$$
\begin{aligned}
& N^{0011}=N^{1100}=-\sin (x)\left[4+k^{2} \operatorname{sech}^{2}\left(\frac{k t+\beta}{2}\right)\right] \\
& N^{0101}=N^{1010}=\sin (x)\left[4+k^{2} \operatorname{sech}^{2}\left(\frac{k t+\beta}{2}\right)\right]
\end{aligned}
$$

Substituting into (15), we find that the only non-vanishing component of the energy density is:

$$
\Omega^{00}=\frac{1}{16 \pi} \sin (x)\left[4+k^{2} \operatorname{sech}^{2}\left(\frac{k t+\beta}{2}\right)\right]
$$


To obtain the total energy, we integrate the energy density $\Omega^{00}$ over the appropriate coordinate ranges (2):

$$
P_{0}=\int_{0}^{\pi} \int_{0}^{4 \pi} \int_{0}^{2 \pi} \Omega^{00} d x d y d z
$$

This yields:

$$
E=P_{0}=\pi\left[4+k^{2} \operatorname{sech}^{2}\left(\frac{k t+\beta}{2}\right)\right]
$$

\section{Using the Technique of Landau and Lifshitz}

Like Papapetrou's procedure, the Landau-Lifshitz energy-momentum complex is symmetric in its indices, permitting conservation laws, such as conservation of angular momentum, to be readily defined.

We define the complex by means of the functions:

$$
L^{i j}=\frac{1}{16 \pi} S_{, k l}^{i j k l}
$$

where the $L^{i 0}$ represent the energy and momentum densities and:

$$
S^{i j k l}=-g\left[g^{i j} g^{k l}-g^{i k} g^{j l}\right]
$$

Substituting into this expression the metric components of the Taub solution (1-5) we find the only relevant non-zero component to be:

$$
S^{0011}=-\frac{k^{2}}{4} \sin ^{2}(x) \operatorname{sech}^{2}\left(\frac{k t+\beta}{2}\right)
$$

From (23), we determine the energy density $L^{00}$ to be:

$$
L^{00}=-\frac{k^{2}}{32 \pi} \cos (2 x) \operatorname{sech}^{2}\left(\frac{k t+\beta}{2}\right)
$$

We now integrate this expression over the full range of coordinates:

$$
P_{0}=\int_{0}^{\pi} \int_{0}^{4 \pi} \int_{0}^{2 \pi} L^{00} d x d y d z
$$

Evaluating this integral, we find the total energy to be:

$$
E=P_{0}=0
$$


So far, we have seen three different results for the total energy, from the Einstein, Papapetrou and Landau-Lifshitz complexes respectively. Perhaps this discrepancy stems from the coordinate system used, which is not quasiCartesian. To obtain coordinate-independent results, we now turn to Møller's energy-momentum complex, specially designed to avoid such issues.

\section{Using Møller's Method}

Møller constructed his complex to transform spatially like a tensor and hence be as free as possible of the choice of coordinate system. Hence, unlike other complexes, it does not favor quasi-Cartesian coordinates. Given that the natural coordinates to define the Taub solution are not quasi-Cartesian, Møller's procedure seems an ideal method to evaluate its gravitational energy.

The quantity Møller developed is defined as:

$$
\Xi_{i}^{k}=\frac{1}{8 \pi} \chi_{i, p}^{k p}
$$

where:

$$
\chi_{i}^{k l}=\sqrt{-g}\left[g_{i p, q}-g_{i q, p}\right] g^{k q} g^{l p}
$$

Inserting the Taub metric components (1-5) we find that for all values of $k$ and $l$ :

$$
\chi_{0}^{k l}=0
$$

Therefore, for all values of $k$ :

$$
\Xi_{0}^{k}=0
$$

Integrating, we obtain the total energy:

$$
E=\int_{0}^{\pi} \int_{0}^{4 \pi} \int_{0}^{2 \pi} \Xi_{0}^{0} d x d y d z=0
$$

Thus, Møller's energy-momentum complex agrees with the prescription of Landau-Lifshitz that the total energy of the vacuum Taub cosmology is zero. We also find that all the momentum components are zero.

\section{Conclusion}


We have investigated the total gravitational energy of the vacuum-filled Taub cosmology using four different energy-momentum complexes: those of Einstein, Papapetrou, Landau-Lifshitz and Møller. Of these, the latter two yielded a value of zero for the total energy of the universe, agreeing with Rosen's conjecture that the total energy of any closed cosmology is zero. The Einstein and Papapetrou complexes, in contrast, yielded non-zero quantities that evolve over time. In our opinion, these two results are suspect because the natural coordinates for the Taub metric, though holonomic, are not quasi-Cartesian, and thus do not fulfill the ideal conditions for these complexes.

We note that our results from the Møller and Landau-Lifshitz complexes agree with earlier work on anisotropic cosmologies, including evaluations of the total energy of the Kasner (Bianchi type I) cosmology for several different cases. We conjecture that the total energy of all vacuum-filled anisotropic cosmologies

is zero. Further calculations, including other Bianchi types, would be needed to confirm this hypothesis.

\section{Acknowledgements}

Thanks to R. T. Jantzen and K. S. Virbhadra for their valuable suggestions. We also wish to thank Timothy Lucas for his assistance. 


\section{References}

[1] A. Einstein, Preuss. Akad. Wiss. Berlin 47, 778 (1915).

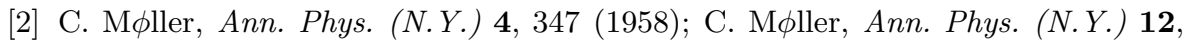
118 (1961).

[3] R.C. Tolman, Phys. Rev. 35, 875 (1930).

[4] A. Papapetrou, Proc. R. Ir. Acad. A 52, 11 (1948).

[5] L. D. Landau and E. M. Lifshitz, The Classical Theory of Fields (Pergamon Press, Oxford, 1962), p. 341.

[6] S. Weinberg, Gravitation and Cosmology: Principles and Applications of the General Theory of Relativity (Wiley, New York, 1972), p. 165.

[7] H. Bondi, Proc. R. Soc. London A427, 249 (1990).

[8] K. S. Virbhadra, Phys. Rev. D41, 1086 (1990); K.S. Virbhadra, Phys. Rev. D42, 1066 (1990); K. S. Virbhadra, Phys. Rev. D42, 2919 (1990).

[9] N. Rosen and K.S. Virbhadra, Gen. Rel. Grav. 25, 429 (1993).

[10] K.S. Virbhadra, Pramana J. Phys. 45, (1995).

[11] J. M. Aguirregabiria, A. Chamorro and K. S. Virbhardra, Gen. Rel. Grav. 28, 1393 (1996).

[12] K.S. Virbhadra, Phys. Rev. D60, 104041 (1999).

[13] S. S. Xulu, "The Energy-Momentum Problem in General Relativity," PhD Dissertation, University of Zululand, South Africa, 2002.

[14] A. Chamorro and K. S. Virbhardra, Int. J. Mod. Phys. D5, 251-256 (1994).

[15] S. S. Xulu, Int. J. Mod. Phys. D7, 773-777 (1998).

[16] I. Radinschi and I. C. Yang, "On the Energy of String Black Holes," New Developments in String Theory Research, ed. Susan A. Grece, (New York: Nova Science, 2005)

[17] E. C. Vagenas, Int. J. Mod. Phys. A18, 5781 (2003).

[18] R. M. Gad, Ap. G S. S. 295, 459 (2005).

[19] S. S. Xulu, Int. J. Theor. Phys. 37, 1773 (2003).

[20] E. C. Vagenas, Int. J. Mod. Phys. A18, 5949 (2003).

[21] E. C. Vagenas, Int. J. Mod. Phys. D14, 578 (2005). 
[22] E. C. Vagenas, Mod. Phys. Lett. A, to appear.

[23] P. Halpern, Mod. Phys. Lett. A21, 495 (2006).

[24] V. C. de Andrade, L. C. T. Guillen and J. G. Pereira, Phys. Rev. Lett. 84, 4533 (2000).

[25] T. T. Bringley, Mod. Phys. Lett. A17, 157 (2002).

[26] R. M. Gad, Ap. ES S. S. 295, 451 (2005).

[27] E. C. Vagenas, Mod. Phys. Lett. A19, 213 (2004).

[28] N. Rosen, Gen. Rel. Grav. 26, 319 (1994).

[29] V.B. Johri, D. Kalligas, G.P. Singh and C.W.F. Everitt, Gen. Rel. Grav. 27, 323 (1995).

[30] F. I. Cooperstock, Found. Phys. 22, 1011 (1992); in Topics in Quantum Gravity and Beyond: Papers in Honor of L. Witten, F. Mansouri and J.J. Scanio, eds. (World Scientific, Singapore, 1993), 201-210; in Relativistic Astrophysics and Cosmology, J. Buitrago, et. al., eds. (World Scientific, Singapore, 1997), 61-72.

[31] N. Banarjee and S. Sen, Pramana J. Phys. 49, 609 (1997).

[32] I. Radinschi, Fizika B 9, 203 (2000).

[33] S.S. Xulu, Int. J. Mod. Phys. A15, 4849 (2000).

[34] O. Aydogdu and M. Salti, Ap. ES S. S. 299, 227 (2005).

[35] M. Salti and A. Havare, Int. J. Mod. Phys. A20, 2169 (2005).

[36] M. Salti, Ap. ES S. S. 299, 159 (2005).

[37] I. Radinschi, Chin. Jour. Phys.39, 231 (2001).

[38] V. A. Belinsky, I. M. Khalatnikov and E. M. Lifshitz, Adv. Phys. 19, 525 (1970).

[39] C. W. Misner, Phys. Rev. Lett. 22, 1071 (1969).

[40] J. D. Barrow, Phys. Rep. 85, 1 (1982).

[41] C. W. Misner and A. H. Taub, Zh. Eksp. Teor. Fiz 55, 233 (1968); English translation: Sov. Phys. JETP 28, 122 (1969).

[42] E. Newman, L. Tamburino and T. Unti, J. Math. Phys. 4, 915 (1963). 